\title{
Adaptive Test for RF/Analog Circuit Using Higher Order Correlations among Measurements
}

\author{
YANJUN LI, University of Electronic Science and Technology of China \\ ENDER YILMAZ, NXP Semiconductor \\ PETE SARSON, Independent \\ SULE OZEV, Arizona State University
}

\begin{abstract}
As process variations increase and devices get more diverse in their behavior, using the same test list for all devices is increasingly inefficient. Methodologies that adapt the test sequence with respect to lot, wafer, or even a device's own behavior help contain the test cost while maintaining test quality. In adaptive test selection approaches, the initial test list, a set of tests that are applied to all devices to learn information, plays a crucial role in the quality outcome. Most adaptive test approaches select this initial list based on fail probability of each test individually. Such a selection approach does not take into account the correlations that exist among various measurements and potentially will lead to the selection of correlated tests. In this work, we propose a new adaptive test algorithm that includes a mathematical model for initial test ordering that takes correlations among measurements into account. The proposed method can be integrated within an existing test flow running in the background to improve not only the test quality but also the test time. Experimental results using four distinct industry circuits and large amounts of measurement data show that the proposed technique outperforms prior approaches considerably.
\end{abstract}

\section{CCS Concepts: • Hardware $\rightarrow$ Analog, mixed-signal and radio frequency test;}

Additional Key Words and Phrases: Initial test list, fail rate, marginality, higher order correlation

\section{ACM Reference format:}

Yanjun Li, Ender Yilmaz, Pete Sarson, and Sule Ozev. 2019. Adaptive Test for RF/Analog Circuit Using Higher Order Correlations among Measurements. ACM Trans. Des. Autom. Electron. Syst. 24, 4, Article 45 (June 2019), 16 pages.

https://doi.org/10.1145/3308566

\section{INTRODUCTION}

For mixed-signal/RF circuits, a large number of measurements are needed to ensure that shipped devices perform with respect to their specifications. Measurement of all specified parameters is prohibitive in terms of test cost. However, since the majority of these parameters are highly correlated, it is possible to contain this cost by judiciously selecting a subset of parameters to be

\footnotetext{
This work is supported by the Fundamental Research Funds for the Central Universities, China. Project NO. ZYGX2016J220 and by National Science Foundation by the award number 1617562.

Author's addresses: Y. Li, University of Electronic Science and Technology of China, School of Automation Engineering, Chengdu, Sichuan, China; email: yjli@uestc.edu.cn; E. Yilmaz, NXP Semiconductor, Austin, TX; email: ender.yilmaz@ nxp.com; P. Sarson, Dialog Semiconductor, Swindon, UK; email: sarsonuk@gmail.com; S. Ozev, Arizona State University, Tempe, AZ; email: sule.ozev@asu.edu.

Permission to make digital or hard copies of all or part of this work for personal or classroom use is granted without fee provided that copies are not made or distributed for profit or commercial advantage and that copies bear this notice and the full citation on the first page. Copyrights for components of this work owned by others than ACM must be honored. Abstracting with credit is permitted. To copy otherwise, or republish, to post on servers or to redistribute to lists, requires prior specific permission and/or a fee. Request permissions from permissions@acm.org.

(C) 2019 Association for Computing Machinery.

1084-4309/2019/06-ART45 \$15.00

https://doi.org/10.1145/3308566
} 
measured and inferring the conformity of the rest of the parameters from available measurements. Traditionally, this selection is done based on a small number of initial measurements and statistical tools that evaluate the potential of these measurements to detect failures [1-4]. This approach has been very effective as static test compaction with the underlying assumption that manufactured devices behave similarly enough to draw effective conclusions for the entire population based on an initial set of samples. However, there is a fundamental trade-off between test time and test quality. With increasing process variations, the similarity assumption no longer holds. The trade-off between test time and test quality becomes unsustainable where one must increase test time continuously to maintain the same quality level. To avoid this pitfall, the test flow must take the diversity in device behavior into account. Adaptive testing is a general term that refers to methods that try to bend the test time-test quality curve by learning from the smaller, localized set of devices or the DUT's own behavior [5-12]. The concept of adaptive test can be applied to learn/optimize within the lot [5], within the wafer [6-8], or within the device under test itself [9-12]. With increasing granularity, there is a better chance to tailor the test, and potentially higher computational effort for learning/optimizing. For statistical test compaction, regardless of whether static or adaptive compaction is used, selecting the correct set of tests to learn from is crucial to increase test quality. This problem is more pronounced for per-device adaptive test, where a small set of measurements has a large influence on the outcome [12]. In per-device adaptive tests, the test list needs to be ordered in a way to enhance information content with respect to the statistical qualities of the device.

Test list ordering has been a research focus for several decades even for static test compaction methods. The most common approach to test list ordering is to model this problem as a set cover problem wherein the goal is to select a minimal subset of existing tests such that all fall-out cases in the sample set can be identified. Although the set cover problem is NP-hard [13], several algorithms have been proposed to solve this problem efficiently. ILP-based formulation [1-2] has been proposed providing near-optimal covers [9]. In Reference [14], Monte Carlo simulations are used to determine the probabilities with which a test covers specifications it was not originally intended to cover [14], along with an ILP model to eliminate unnecessary tests. Heuristic approaches [3-4] [15] can handle large test sets where ILP-formulation is no longer computationally feasible. Once a test set is identified, test set re-ordering [15-17] is proposed to further reduce the amortized test time by first applying the tests most likely to fail. In References [10-11] a heuristic method is used for solving the set cover problem to order the initial test list. Most set-cover-based test compaction methods use only fail data and disregard correlations among the specifications for which at least one device has failed. In Reference [18], the authors propose to continuously monitor the effectiveness of tests for a given DUT and the test order is modified sequentially.

A number of static test compaction methods take correlations among parameters into account when selecting which tests to apply [19,22]. A two-class support vector machine is used to model the correlation between pass/fail patterns of devices [19]. An SVM trained in this fashion may pass or fail devices based on incomplete information and thus is subject to both yield loss and test escapes. In Reference [22], tests are selected both based on their process capability index and correlations among them. In References [20-21], the goal is to select easier tests and infer the pass/fail status of more complex tests. Pareto analysis was used to determine the optimal test set [6], and a binominal distribution function is used to estimate the fault probability. Correlation between test suites was analyzed using canonical correlation [23], and a genetic algorithm was applied to optimize the test time reduction.

In the per-device adaptive test domain, test ordering has been based on coverage, marginality, and standard deviation of the measurement distribution [12]. In Reference [12], the authors present an adaptive test flow that skips tests based on earlier measurements. The learning process is based on incremental mapping to enable continuous background learning. 
Since per-device adaptive testing provides the best granularity and quality/time trade-off, it is our focus in this work. We present a mathematical model and an algorithm for test list ordering to improve per-device adaptive testing. The main purpose of ordering the test list is to ensure that the statistical estimator gains adequate information in as few tests as possible. Moreover, since defective devices and process shifts may alter some of the learned information, tests with more information content need to be applied before a decision can be made on the DUT. We use fail rate, marginality, and higher-order correlations in ordering the test list to ensure that maximum information content is attained early in the test process. To model higher-order correlations, the sample set is reconstructed as linear combinations of uncorrelated variables. A modified QR decomposition using Givens rotation is implemented to order the initial test list. The proposed adaptive test method yields better test quality and test time trade-off for three industry circuits, and performs similarly for a fourth industry circuit compared to the best results reported in prior work [12].

\section{BACKGROUND}

In general, an adaptive test flow is composed of two stages: the static stage and the test stage, as shown in Figure 1. In the static stage, the good devices of the sample set are used to train the specification model, the failed devices are used to order the test list, and the outliers are used to train the outlier filter. Then, in the test stage, the specification model is updated according to the measurement of the tests applied and then is used to predict the test about to apply. In our work, the static modeling is similar to that of Reference [12], where a joint probability distribution is defined by Equation (1):

$$
\begin{gathered}
\int_{-\infty}^{+\infty} K(x)=1 \\
\operatorname{PDF}(\mathbf{s})=\frac{1}{n \prod_{j=1}^{n} h_{j}} \sum_{i=1}^{n} \omega_{i} \prod_{j}^{M} K\left(\frac{s_{j}-\mathrm{S}_{i, j}}{h_{j}}\right)
\end{gathered}
$$

In Equation (1), $K(x)$ is the normal kernel, $\mathbf{P D F}(\mathbf{s})$ is the estimated joint probability distribution function, $\boldsymbol{s}$ is the specification vector of the joint PDF (JPDF), $\mathbf{S}_{i}$ is a vector containing specifications of the $i$ th device in the training set, $h_{j}$ is the kernel width of the $j$ th kernel, $\omega_{i}$ is the kernel weight, $n$ is the size of the training set, and $M$ is the number of the specifications. For best fit, the kernel width $h_{j}$ is set according to Equation (3):

$$
h_{j}=\left(\frac{1}{d+2}\right)^{\frac{1}{d+4}} \sigma_{j} n^{-\frac{1}{d+4}},
$$

where $\sigma_{j}$ is the standard deviation of the $j$ th specification parameters and $d$ is the number of dimensions (non-measured parameters).

Kernel-based PDF estimation technique simply superimposes kernels on each training sample $\left(S_{i}\right)$. It can be viewed as the weighted summation of localized kernel of each training sample. As each training sample is treated equally, $\omega_{i}$ is set to 1 at the static stage.

Figure 1 shows the flow of per-device adaptive test. During the test stage, at the beginning of each device's test process, the JPDF and the initial test list are reloaded. The tests are fetched oneby-one according to the sequence of initial test list. Each test in the list $\left(T_{j}\right)$ is evaluated based on its fail probability, (Pfail). The Pfail yields the conditional cumulative density function, which is the fail probability of the current test according to the JPDF, and measured test results up to that point. If the fail probability is lower than a pre-determined threshold, then the test, $T_{j}$, will be skipped, saving time. Otherwise, it will be applied to the current DUT. If applied, then the result 


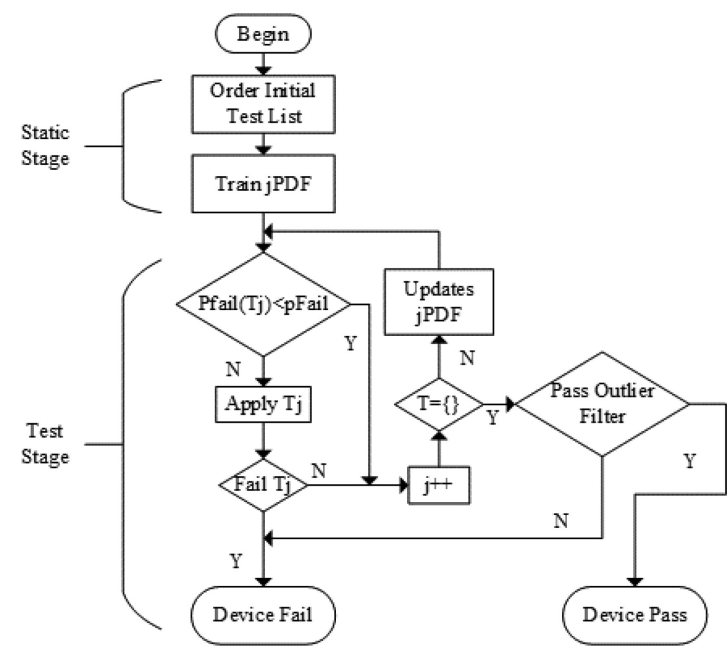

Fig. 1. Adaptive test process.

of $T_{j}$ is used to update the JPDF according to Equations (4) and (5):

$$
\begin{gathered}
\hat{\operatorname{PDF}}\left(s_{j}\right)=\frac{1}{\left(\sum \omega^{\prime}{ }_{i}\right) \prod h_{j}} \sum_{i} \omega_{i}^{\prime} \prod_{s_{j} \in T} K\left(\frac{s_{j}-S_{i, j}}{h_{j}}\right), \\
\omega^{\prime}{ }_{i}=\left[\prod_{m_{j} \in T^{\prime}} K\left(\frac{m_{j}-S_{i, j}}{\alpha h_{j}}\right)\right] \omega_{i},
\end{gathered}
$$

where $\omega_{i}^{\prime}$ is the updated weight of $i$ th kernel, $T^{\prime}$ is the set of conducted tests, and $\alpha$ is the kernel penalization coefficient. The updated weights $\left(\omega_{i}^{\prime}\right)$ are normalized such that they sum up to $n$. During this process, the weight of each kernel is updated according to Equation (5), based on the distance of the kernel from the test result. Thus, the JPDF in Equation (4) is localized to the current DUT.

On one hand, time-saving is the main purpose of the adaptive test. Although the tests are treated individually without considering that in reality they are grouped according to the test configurations, the majority of the tests are low-frequency measurements, where the test time equals or exceeds the test set-up time. Thus, skipping some tests could save the test time overall. On the other hand, computation overhead for the model updating and the test prediction should not be added to the critical path of the test process. Figure 2 illustrates the pipelined test methodology, as the measurement for Test [i+1], the data processing for Test [i], as well as the prediction for Test $[i+2]$ are performed concurrently.

Since defects alter the relations among circuit parameters, there is a chance that a defective circuit will pass applied tests but will fail skipped tests. Thus, as a final step, it is necessary that conformity with respect to expected behavior is confirmed based on available measurements. An outlier filter is used for this purpose. This filter applies a distance gauge as presented in Equations (6) and (7) to identify the outlier devices:

$$
D_{j}=-\log \left\{\frac{1}{N} \sum_{k}^{N} K_{j}\left(\frac{s_{i, j}-\mu_{i, j}}{h_{j}}\right)\right\},
$$




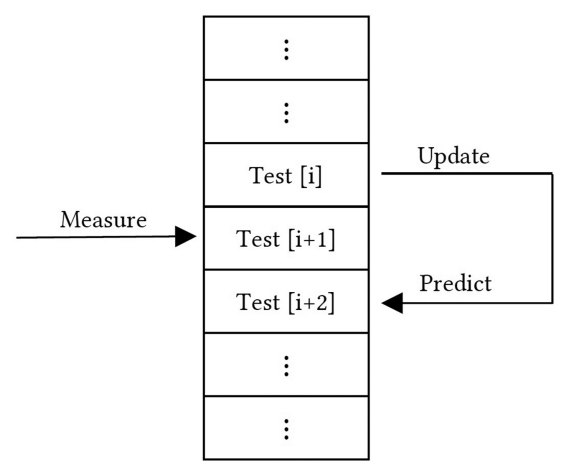

Fig. 2. Pipelined time schedule.

$$
D_{\{j\}}\left(s_{i}\right)=\sum_{\{j\}} D_{j}\left(s_{j}\right)
$$

where $D_{j}$ is the distance of the device parameter vector $s_{i}$ in $j$ th dimension. $D_{\{j\}}$ combines the distances defined by $D_{j}$ in parameter set $\{j\}$ and yields a scalar number that is the distance of $s_{i}$ from the nominal. A boundary at a high quantile such as $99.9 \%$ was used to separate the outliers. Outlier-free DUTs will pass the test, and the outliers will be retested with the full test suite. If the outliers fail the full tests, then they are merged with the outliers from the sample set to relearning the outlier filter for anti-process-shifting purpose. For the same purpose, devices passing the full test are the candidates for relearning the JPDF, and the ones failing the full test are the candidates for reordering the test list in Reference [12]. However, in the proposed, this anti-process-shifting methodology is not applied, because the results are not much different for the four circuits data.

In this section, we introduce the methodology of the adaptive test flow, which is described in Reference [12]. In the static stage, the sample set is used to train the specification model, order the initial test list, and train the outlier filter. In the test stage, the JPDF is reloaded for each DUT and is updated after every measurement for every unique device on every single test site, which means this adaptive test flow is compatible with multi-site testing. In the per-device adaptive test flow, the initial test list is the same for all DUTs and is determined by the statistical information of the training sample set. In References [10-12], the initial test list is ordered considering the covering test condition, marginality condition, and specification tightness condition. In Reference [23], tests by an outlier filter are placed in the initial test list to reduce the test time. In this article, we take a different approach for test list ordering. We model higher-order correlations among the specifications and use these correlations to order the test list with the goal of obtaining as much non-overlapping information as possible.

\section{CONDITIONS FOR INITIAL TEST LIST ORDERING}

It has been shown in prior work that the order of the initial test list is a crucial factor affecting test quality and the test time. In test list ordering, several criteria, such as selecting tests that cover all failures in the given characterization set or selecting marginally passing tests, are typically used. However, for most prior approaches, test list ordering is a uni-dimensional process, i.e., the tests are selected solely based on their statistical parameters, and correlations among tests are not factored into the selection process.

In contrast, the proposed test list ordering algorithm takes into account two factors, namely the fail probability of each test and the incremental information content of each test when higher-order correlations are taken into account. 


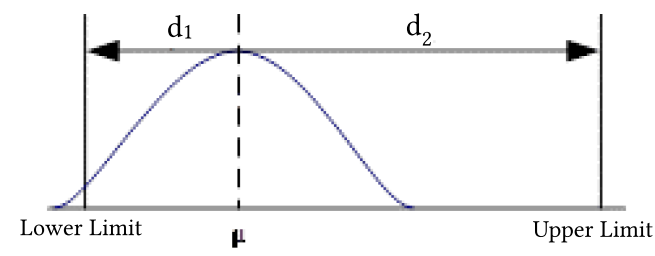

Fig. 3. Marginality of specification.

\subsection{Fail Rate}

Fail rate of a test is defined as the ratio of the number of circuit instances from the characterization set that fail that test to the number of overall circuit samples. This information has been heavily used in prior work for test selection [12]. Roughly half of the fails are due to catastrophic defects (e.g., continuity fails). In such cases, the devices are taken out of consideration, as the measurement data is incomplete in training. The other half of the fails are parametric ones, with which we count the fail rate of the tests. However, using only fail rate does not take into account existing correlations among circuit parameters.

\subsection{Marginality Condition}

The marginality of a test is defined based on its $C_{p k}$ value, which is the ratio of the smallest distance of the test average from the test limits divided by the standard deviation of the measurements. As an example, the marginality of the specification shown in Figure 3 is $d_{1} / \sigma$. Marginality represents the fail probability of a test, which is non-zero even if none of the current samples have failed that test.

Marginality is a quantitative gauge for fail probability, but it has a similar disadvantage as fail rate, because the correlations among the tests are not taken into account. For instance if the marginality of tests are ordered as $\mathrm{T} 1<\mathrm{T} 2<\mathrm{T} 3$, the resulting test list will have the reverse order as $\{\mathrm{T} 1, \mathrm{~T} 2, \mathrm{~T} 3\}$. However, if $\mathrm{T} 1$ and $\mathrm{T} 2$ are highly correlated while $\mathrm{T} 1$ and $\mathrm{T} 3$ are not, the optimum test list may be $\{\mathrm{T} 1, \mathrm{~T} 3, \mathrm{~T} 2\}$. For the tests not failed in the sample set, the test list is ordered considering the marginality and the correlations among the test simultaneously.

\subsection{Correlation Condition}

Our goal in ordering the test list is to obtain as much information as early as possible. Since we are trying to maximize information content, we refer to Shannon information theory [24] to define useful information. For simplicity, let us consider a bi-variate case. We denote the probability function of the $i$ th test with $p(i)$, the probability function of the $j$ th test with $p(j)$, and the joint probability function of the $i$ th and the $j$ th tests, with $p(i, j)$. The amount of the information associated with the two tests can be measured by the joint entropy of them, as in Equation (8):

$$
H(i, j)=-E_{p(i, j)} \log p(i, j),
$$

where $E$ denotes the mathematical expectation. As circuit parameters are correlated, one test contains information about another, which can be measured by the mutual information $I(i: j)$, as in Equation (9):

$$
I(i: j)=E_{p(i, j)} \log \frac{p(i, j)}{p(i) p(j)} .
$$

Additionally, the relation between mutual information and joint information is given as in Equation (10), where $H(i)$ and $H(j)$ denote the information entropy of test $i$ and test $j$, respectively. Equation (10) indicates that the information of two tests is no more than the sum of the information 


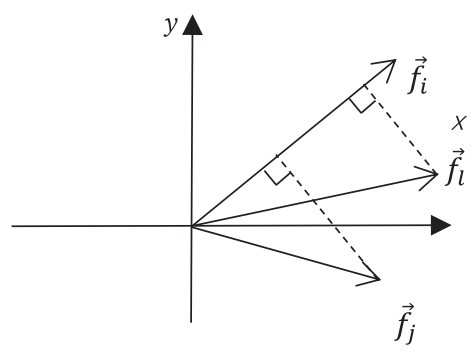

Fig. 4. Weighted fail rate in Step 2.

of each individual test. If test $i$ and test $j$ are independent, then the mutual information of them equals to zero, as in Equation (11):

$$
H(i, j)=H(i)+H(j)-I(i: j) .
$$

Thus, the joint information has its maximum value, as in Equation (11):

$$
H(i, j)=H(i)+H(j) .
$$

Conversely, the information content of two correlated tests is lower compared to the case where they are uncorrelated. From this perspective, test ordering should aim to include as many uncorrelated tests as possible early on in the test flow.

\section{METHOD FOR INITIAL TEST LIST ORDERING}

In the proposed method, fail rate, marginality, and correlation conditions are taken into account simultaneously to order the initial test list.

\subsection{Ordering Strategy}

In the proposed test list ordering methodology, the fail rate and marginality serve as ordering conditions, and higher-order correlations serve as weight factors. The initial test list has two parts: the top part with the failed tests in the sample set and the later part with the un-failed tests. The proposed ordering strategy is explained in the following steps:

1. Place the test with the highest fail rate at the top of the list: The test that fails the highest number of devices from the characterization set is applied first.

2. Iteratively, place the test with highest weighted fail rate next in the list of the top part.

3. Order the later part based on marginality using the same weighing approach.

Weighted fail rate: The weighted fail rate is defined for two tests as in Equation (12), where, $\widehat{f}_{j}$ is the weighted fail rate and $f_{j}$ is the original fail rate of the $j$ th test, $p_{i, j}$ is the correlation between the $j$ th test and the $i$ th test:

$$
\hat{f}_{j}=f_{j} \sqrt{1-\rho_{i, j}^{2}} \quad(j=1,2, \ldots, p \text { and } j \neq i) .
$$

The concept of weighted fail rate is demonstrated in Figure 4. Suppose tests are represented as vectors in an $\mathrm{M}$-dimensional space and the cosine of the angle between the $i$ th test vector and the $j$ th test vector represents the correlation $\rho_{i, j}$ between them. The length of vector $\vec{f}_{i}$ is the original fail rate of test $i$. The weighted fail rate, $\widehat{f}_{j}$, is the component of vector $\vec{f}_{j}$ perpendicular to vector $\vec{f}_{i}$. The component of $\vec{f}_{j}$ parallel to $\vec{f}_{i}$ fully correlated to $\vec{f}_{i}$, thus, it has no contribution to the 


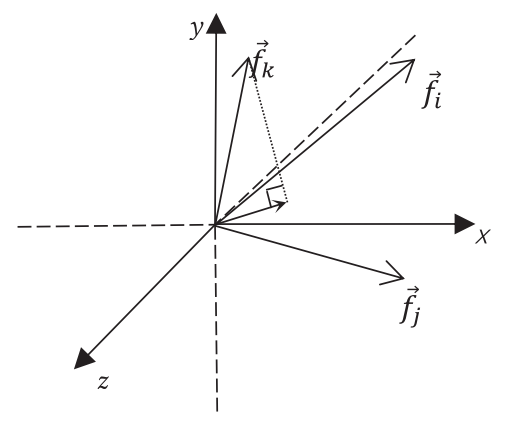

Fig. 5. Weighted fail rate in Step 3.

information content. Suppose in Figure 4, the test $i$ has already been selected. To select the next test, we evaluate the relative information content of tests $j$ and $k$. Although the length of vector $\vec{f}_{j}$ is smaller than that of vector $\vec{f}_{l}$, it is selected as the second test because it has a bigger component perpendicular to $\vec{f}_{i}$ than $\vec{f}_{l}$.

The definition of weighted fail rate can be generalized as in Equation (13) for higher dimensions:

$$
\hat{f}_{k}=f_{k} \sqrt{1-\rho_{i j, k}^{2}}
$$

Continuing along the same example, suppose that tests $i$ and $j$ are already selected. Figure 5 illustrates how an additional test can be evaluated in terms of information content. In Figure 5 , $\rho_{i j, k}$ defines the correlation between test $k$ and the plane formed by tests $i$ and $j$. If we denote $\theta_{i j, k}$ as the angle between vector $\overrightarrow{f_{k}}$ and the plane spanned by vectors $\vec{f}_{i}$ and $\vec{f}_{j}$, then $\rho_{i j, k}$ equals to the cosine of $\theta_{i j, k}$.

Marginality: The marginality condition of each test can also be weighted by the correlation, as in Step 2, to order the remaining tests.

The major challenge in computing the weighing factors is defining the correlations for a large test set. Next, we will discuss our novel methodology for modeling and using correlations as weighing factors.

\subsection{Sample Set Reconstruction}

To calculate the weighing coefficients, we first express each measurement in terms of uncorrelated basis functions. Let us denote centered specifications of circuit samples in matrix form as in Equation (14), where the column vector $\mathbf{x}_{i}=\left[\begin{array}{lll}x_{1 i} & x_{2 i} \ldots x_{n i}\end{array}\right]^{\prime}$ is the $i$ th centered test results with $\mathrm{n}$ samples, i.e., each $x_{i}$ is the result of the measurement minus its empirical mean value:

$$
\mathrm{X}=\left[\begin{array}{llll}
\mathbf{x}_{1} & \mathbf{x}_{2} & \cdots & \mathbf{x}_{p}
\end{array}\right]
$$

Thus, $\mathbf{X}$ is a $p \times n$ matrix with $n>p$. By singular value decomposition, the matrix $\mathbf{X}$ can be decomposed as in Equation (15), where $\mathbf{U}=\left[\mathbf{u}_{1} \ldots \mathbf{u}_{p} \ldots \mathbf{u}_{n}\right]$ is an $n \times n$ orthogonal matrix, $\mathbf{V}$ is an $p \times p$ orthogonal matrix, and $\Sigma$ is the singular value matrix, given in Equation (16):

$$
\mathbf{X}=\mathbf{U} \Sigma \mathbf{V}^{\prime}
$$




$$
\Sigma=\left[\begin{array}{cccccc}
\sigma_{1} & 0 & 0 & 0 & \cdots & 0 \\
0 & \ddots & 0 & \vdots & \cdots & 0 \\
0 & 0 & \sigma_{p} & 0 & \cdots & 0 \\
0 & \cdots & 0 & 0 & \ddots & 0 \\
0 & \cdots & 0 & 0 & \cdots & 0
\end{array}\right] .
$$

In Equation (16), $\sigma_{1} \geq \sigma_{2} \geq \cdots \geq \sigma_{p}>0$. Ignoring the zero entries, $\mathbf{U}$ and $\Sigma$ can be tailored and Equation (15) can be modified, as in Equation (17):

$$
\mathbf{X}=\left[\begin{array}{lll}
\mathbf{u}_{1} \cdots & \mathbf{u}_{p}
\end{array}\right]\left[\begin{array}{ccc}
\sigma_{1} & 0 & 0 \\
0 & \ddots & 0 \\
0 & 0 & \sigma_{p}
\end{array}\right] \mathbf{V}^{\prime}=\mathbf{U}_{p} \sum_{p} \mathbf{V}^{\prime}
$$

In Equation (17), $\mathbf{U}_{p}=\left[\mathbf{u}_{1} \ldots \mathbf{u}_{p} \ldots \mathbf{u}_{p}\right]$ and

$$
\Sigma_{p}=\left[\begin{array}{lll}
\sigma_{1} & 0 & 0 \\
0 & \ddots & 0 \\
0 & 0 & \sigma_{p}
\end{array}\right]
$$

Note that $\mathbf{u}_{i}(i=1, \ldots, p)$ are mutually uncorrelated, and each $\mathbf{u}_{i}$ has the same variance $\sigma_{\mathbf{u}_{1}}^{2}=\cdots=$ $\sigma_{\mathbf{u}_{p}}^{2}=\sigma_{\mathbf{u}}^{2}$.

We denote the weight matrix from $\mathbf{U}_{p}$ to $\mathbf{X}$ as $\mathbf{W}$, as in Equation (18):

$$
\mathrm{X}=\mathrm{U}_{p} \mathbf{W} \text {. }
$$

The weight matrix can be obtained by Equation (19):

$$
\mathbf{W}=\sum_{p} \mathbf{V}^{\prime}=\left[\begin{array}{ccc}
w_{11} & \cdots & w_{1 p} \\
\vdots & \ddots & \vdots \\
w_{p 1} & \cdots & w_{p p}
\end{array}\right]=\left[\mathbf{w}_{1} \cdots \mathbf{w}_{p}\right]
$$

The sample set can be expressed as in Equation (20) and Equation (21):

$$
\begin{gathered}
{\left[\begin{array}{lll}
\mathbf{x}_{1} & \cdots & \mathbf{x}_{p}
\end{array}\right]=\left[\begin{array}{lll}
\mathbf{u}_{1} & \cdots & \mathbf{u}_{p}
\end{array}\right]\left[\begin{array}{ccc}
w_{11} & \cdots & w_{1 p} \\
\vdots & \ddots & \vdots \\
w_{p 1} & \cdots & w_{p p}
\end{array}\right],} \\
\mathbf{x}_{i}=\sum_{k=1}^{p} w_{k i} \mathbf{u}_{k} .
\end{gathered}
$$

The correlation coefficient between two vectors $\mathbf{x}_{i}$ and $\mathbf{x}_{j}$ can be expressed as in Equation (22), where $\operatorname{cov}\left(\mathbf{x}_{i}, \mathbf{x}_{j}\right)$ denotes the covariance of $\mathbf{x}_{i}$ and $\mathbf{x}_{j}$, and $\sigma_{i}$ and $\sigma_{j}$ denote the standard variations of $\mathbf{x}_{i}$ and $\mathbf{x}_{j}$ :

$$
\rho_{i, j}=\frac{\operatorname{cov}\left(\mathbf{x}_{i}, \mathbf{x}_{j}\right)}{\sigma_{i} \sigma_{j}} .
$$

Thus, the correlation between $\mathbf{x}_{i}$ and $\mathbf{x}_{j}$ can be calculated as in Equation (23):

$$
\rho_{i, j}=\frac{\sigma_{\mathbf{u}}^{2} \sum_{k=1}^{p} w_{k i} w_{k j}}{\sqrt{\sigma_{\mathbf{u}}^{2} \sum_{k=1}^{p} w_{k i}^{2}} \sqrt{\sigma_{\mathbf{u}}^{2} \sum_{k=1}^{p} w_{k j}^{2}}}=\frac{\sum_{k=1}^{p} w_{k i} w_{k j}}{\sqrt{\sum_{k=1}^{p} w_{k i}^{2}} \sqrt{\sum_{k=1}^{p} w_{k j}^{2}}} .
$$


Equation (23) indicates that the correlation between $\mathbf{x}_{i}$ and $\mathbf{x}_{j}$ is determined by vectors $\mathbf{w}_{i}$ and $\mathbf{w}_{j}$ or by the cosine of the angle $\theta_{i, j}$ between vector $\mathbf{w}_{i}$ and vector $\mathbf{w}_{j}$, as in Equation (24):

$$
\cos \theta_{i, j}=\frac{\sum_{k=1}^{p} w_{k i} w_{k j}}{\sqrt{\sum_{k=1}^{p} w_{k i}^{2}} \sqrt{\sum_{k=1}^{p} w_{k j}^{2}}}=\rho_{i, j} .
$$

Thus, the weighted fail rate of Equation (12) can be calculated as in Equation (25):

$$
\hat{f}_{j}=f_{j} \sqrt{1-\rho_{i, j}^{2}}=f_{j} \sin \theta_{i, j} \quad(j=1,2, \ldots, p \text { and } j \neq i) .
$$

Similarly, the weighted fail rate of Equation (13) can be calculated as in Equation (26), where $\theta_{i j, k}$ is the angle between vector $\mathbf{w}_{k}$ and the plane spanned by vector $\mathbf{w}_{i}$ and vector $\mathbf{w}_{j}$ :

$$
\hat{f}_{k}=f_{k} \sqrt{1-\rho_{i j, k}^{2}}=f_{k} \sin \theta_{i j, k} .
$$

Note that Equation (26) can be extended to dimensions more than 3. By means of the sample set reconstruction, the specifications are represented by linear combinations of uncorrelated variables, as shown in Equation (21). At each step, the correlation that we are interested in is the cosine of the angle between the corresponding vector and the hyperplane spanned by the vectors concerned in matrix $\mathbf{W}$. The weighted fail rate can be computed by multiplying the sine of the angle between the vector and the hyperplane spanned by the vectors corresponding to the tests already in the initial test list.

\subsection{Ordering Algorithm}

For simplicity, the column vector matrix $\mathbf{W}$ can be normalized as in Equation (27), without changing the direction of the column vector, hence without changing the angle between the column vectors:

$$
\tilde{\mathbf{w}}_{i}=\frac{\mathbf{w}_{i}}{\sqrt{\mathbf{w}_{i}^{\prime} \mathbf{w}_{i}}} \quad(i=1,2, \ldots, p) .
$$

The fail rate of each parameter is multiplied to the corresponding $\tilde{\mathbf{w}}_{i}$ as in Equation (28), where $l$ represents the number of tests that fail at least one device from the sample set. Thus, the length of each $\hat{\mathbf{w}}_{i}$ is the original fail rate of each parameter:

$$
\hat{\mathbf{w}}_{i}=f_{i} \tilde{\mathbf{w}}_{i} \quad(i=1,2, \ldots, l) .
$$

We define the fail matrix as in Equation (29):

$$
\hat{\mathbf{W}}=\left[\begin{array}{llll}
\hat{\mathbf{w}}_{1} & \hat{\mathbf{w}}_{2} & \cdots & \hat{\mathbf{w}}_{l}
\end{array}\right] .
$$

Note that $\hat{\mathbf{W}}$ is a $p \times l$ matrix. We implement a modified $\mathrm{QR}$ decomposition using Givens rotation [25] to order the initial test list.

4.3.1 Find the First Test to Apply. According to the ordering strategy, the specification with the largest fail rate should be applied first. Thus, we place the vector $\hat{\mathbf{w}}_{i}$ with the largest length on top of the test list. Without loss of generality, we assume $\hat{\mathbf{w}}_{1}$ is chosen as the first vector and rewrite the fail matrix, as in Equation (30):

$$
\hat{\mathbf{W}}=\left[\begin{array}{cccc}
\hat{w}_{11} & \hat{w}_{12} & \cdots & \hat{w}_{1 l} \\
\hat{w}_{21} & \hat{w}_{22} & \cdots & \hat{w}_{2 l} \\
\vdots & \vdots & \ddots & \vdots \\
\hat{w}_{p 1} & \hat{w}_{p 2} & \cdots & \hat{w}_{p l}
\end{array}\right] .
$$


Then, we need to find the vector with the biggest component perpendicular to the chosen vectors, as we discussed in 4.1. For this purpose, Givens rotation is applied to $\hat{\mathbf{W}}$ to make $\hat{\mathbf{w}}_{1}^{1}$ into a column vector with 0 elements except the first one, as in Equation (31), where $\mathbf{Q}_{1}$ is an orthogonal matrix, which means that the rotation will not change the length of each column vector, as well as the angle between each pair of column vectors:

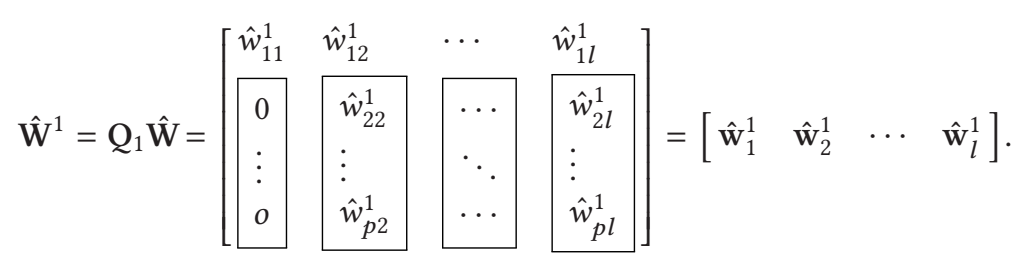

We can see that the components in frames of each vector from $\hat{\mathbf{w}}_{2}^{1}$ to $\hat{\mathbf{w}}_{l}^{1}$ are perpendicular to $\hat{\mathbf{w}}_{1}^{1}$. In other words, the weighted fail rate defined in Section 4.1 for the $i$ th parameter is as in Equation (32):

$$
\hat{f}_{i}=\sqrt{\left(\hat{w}_{2 i}^{1}\right)^{2}+\left(\hat{w}_{3 i}^{1}\right)^{2}+\cdots+\left(\hat{w}_{p i}^{1}\right)^{2}} \quad(i=2, \ldots, l) .
$$

4.3.2 Order the Rest Tests. As discussed in 4.1, the ordering strategy is to find a test with the largest weighted fail rate in every ordering step. A modified $\mathrm{QR}$ decomposition using Givens rotation is applied to the fail matrix as the following steps:

Step 1: Find the vector according to the constraint given in Equation (33):

$$
\hat{f}_{j}=\max \left\{\hat{f}_{i}, \quad i=2,3, \ldots, l\right\} .
$$

Without loss of genericity, suppose from $\hat{\mathbf{w}}_{2}^{1}$ to $\hat{\mathbf{w}}_{l}^{1}$, we find $\hat{\mathbf{w}}_{j}^{1}$ according to Equation (33), which means that $\hat{\mathbf{w}}_{j}^{1}$ has the largest weighted fail rate.

Step 2: Exchange the positions of $\hat{\mathbf{w}}_{2}^{1}$ and $\hat{\mathbf{w}}_{j}^{1}$, as in Equation (34):

$$
\hat{\mathbf{W}}^{1, P}=\hat{\mathbf{W}}^{1} \mathbf{P}^{1},
$$

where $\hat{\mathbf{W}}^{1, P}$ is the matrix after the permutation of $\hat{\mathbf{W}}^{1}$ with $\hat{\mathbf{w}}_{2}^{1}$ and $\hat{\mathbf{w}}_{j}^{1}$ exchanging their positions, and $\mathbf{P}^{1}$ is an orthogonal matrix, as in Equation (35):

$$
\mathbf{P}^{1}=\left[\begin{array}{cccccc}
1 & 0 & \cdots & 0 & \cdots & 0 \\
0 & 0 & \cdots & p_{2, j}^{1}=1 & \cdots & 0 \\
\vdots & \vdots & \ddots & \vdots & \ddots & \vdots \\
0 & p_{l, 2}^{1}=1 & \cdots & 0 & \cdots & 0 \\
\vdots & \vdots & \ddots & \vdots & \ddots & \vdots \\
0 & 0 & \cdots & 0 & \cdots & 1
\end{array}\right]
$$

Step 3: Apply Givens rotations to $\hat{\mathbf{W}}^{1, P}$, as in Equation (36):

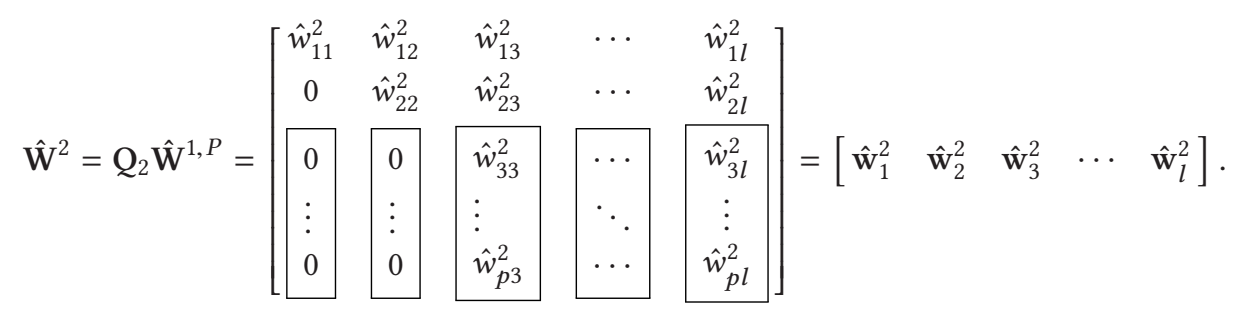




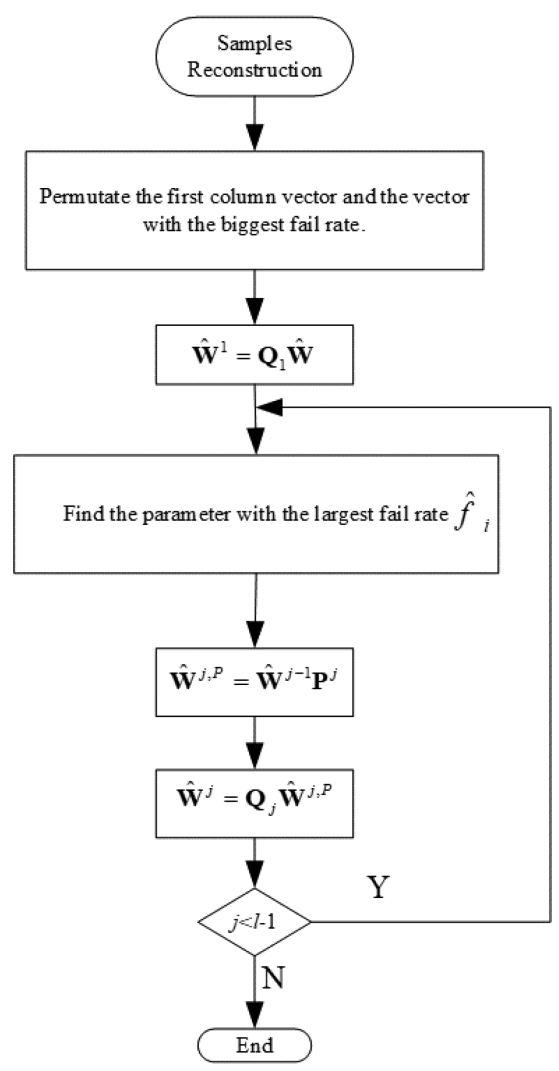

Fig. 6. Initial test list ordering flow.

After this step, only the first two components of $\hat{\mathbf{w}}_{2}^{2}$ are non-zero. We can see that the components in frames of each vector from $\hat{\mathbf{w}}_{3}^{2}$ to $\hat{\mathbf{w}}_{l}^{2}$ are perpendicular to the plane spanned by $\hat{\mathbf{w}}_{1}^{2}$ and $\hat{\mathbf{w}}_{2}^{2}$. Thus, the weighted fail rate for the $i$ th parameter is given in Equation (37):

$$
\hat{f}_{i}=\sqrt{\left(\hat{w}_{3 i}^{2}\right)^{2}+\left(\hat{w}_{4 i}^{2}\right)^{2}+\cdots+\left(\hat{w}_{p i}^{2}\right)^{2}} \quad(i=3, \ldots, l) .
$$

Also, the vector with the largest weighted fail rate is found, as in Equation (38):

$$
\hat{f}_{k}=\max \left\{\hat{f}_{i}, \quad i=3,4, \ldots, l\right\} .
$$

Step 4: Repeat Step 1 to Step 3. At each iteration, we find a vector has the largest component perpendicular to the hyperplane spanned by the previous test vectors. With Givens rotation, it is convenient to find the test parameter with the largest weighted fail rate. The steps of test reordering are presented in Figure 6. The marginality condition is handled in a similar way.

\section{RESULTS}

The proposed initial test list ordering algorithm is integrated into the test method in Reference [12]. Industry data from four circuits are used to evaluate the efficacy of the proposed algorithm. The first circuit is an RF circuit with 42 specifications and many building blocks, and $30 \mathrm{~K}$ data is collected over six months. The second circuit is a mixed-signal circuit with 70 specifications, and 50K 
Table 1. Comparison for the Four Circuits

\begin{tabular}{|l|c|c|c|c|}
\hline & \multicolumn{2}{|c|}{ DPPM } & \multicolumn{2}{c|}{ Number of Tests/All } \\
\hline & Proposed & Reference [12] & Proposed & Reference [12] \\
\hline Circuit 1 & 35.7 & 35.7 & $14.5 / 42$ & $14.9 / 42$ \\
\hline Circuit 2 & 145.8 & 806.3 & $17.8 / 70$ & $20.0 / 70$ \\
\hline Circuit 3 & 83.3 & 105 & $75.7 / 264$ & $80 / 264$ \\
\hline Circuit 4 & 78 & 117 & $24.5 / 57$ & $26.4 / 57$ \\
\hline
\end{tabular}

Table 2. Escaped Tests Comparison for Circuit 1

\begin{tabular}{|l|c|c|c|}
\hline \multirow{4}{*}{ Reference [12] } & Test No. & 26 & 27 \\
\cline { 2 - 4 } & Escaped Rate & 1 & 2 \\
\cline { 2 - 4 } & Subgroup Length & 13 & 3 \\
\hline \multirow{3}{*}{ Proposed } & Test No. & 10 & \\
\cline { 2 - 4 } & Escaped Rate & 1 & \\
\cline { 2 - 4 } & Subgroup Length & 13 & \\
\hline
\end{tabular}

data from 9 wafers is collected over a six-month period. The third circuit is a large-scale circuit with 264 specifications, and $50 \mathrm{~K}$ data is collected over nine months. The fourth circuit is an EEPROM with 57 analog specifications selected to verify our method, and 1,002K data from 376 wafers/15 lots is collected over nine months. For all the four circuits, $2 \mathrm{~K}$ data is used as the sample set; $28 \mathrm{~K}$ data for the first circuit and $48 \mathrm{~K}$ data for the second and third circuits, as well as $1 \mathrm{M}$ data for the fourth circuit, are used to verify the method.

Test escape and average Number of Tests are two important gauges in circuit testing that are generally in trade-off with one another. We compare the results of the improved per-device adaptive test approach to that of Reference [12], which is the best reported result based on industry data so far. Moreover, the rest of the adaptive test algorithm is identical, hence any improvement in test metrics is due to the proposed test list ordering approach. We use DPPM as test escape per million samples and the average Number of Tests applied in the test process. Table 1 shows the comparison between the proposed method and the method in Reference [12]. Because the partial initial test list in Reference [12] is randomly generated, the results from the method in Reference [12] shown in Table 1 are the average values of 10 runs for each circuit. The proposed method orders the initial test list by systemically analyzing the sample data. Thus, the initial test list for a specific sample set does not change from run to run. With the proposed test-ordering algorithm, tests with higher fail rate are applied first, which saves the test time. Moreover, with the algorithm, less correlated tests are applied prior, which obtains more incremental information of each DUT. Thus, it achieves overall lower DPPM and lower Number of Tests for the adaptive test method in Reference [12].

\subsection{RF Circuit}

First, we verify the proposed method on an RF circuit production data with 42 specifications. The data set has $30 \mathrm{~K}$ samples with 42 specifications; $2 \mathrm{~K}$ samples out of this data set are selected as the training set and the rest of the data are used to compute the DPPM and the Number of Tests. Table 1 shows that the proposed method has similar DPPM and Number of Tests as the average values of the method in Reference [12].

Table 2 shows the comparison of escaped tests for the two methods. Although the two methods result in similar DPPMs, the test escapes are due to different tests. 
Table 3. Escaped Tests Comparison for Circuit 2

\begin{tabular}{|c|c|c|c|c|c|c|c|c|c|c|}
\hline \multirow{3}{*}{ Reference [12] } & Test No. & 38 & 58 & 59 & 61 & 63 & 65 & 41 & 46 & 68 \\
\hline & Escaped Rate & 1 & 20 & 20 & 20 & 20 & 20 & 5 & 35 & 4 \\
\hline & Subgroup Length & \multicolumn{6}{|c|}{28} & 15 & 2 & 1 \\
\hline \multirow{3}{*}{ Proposed } & Test No. & 38 & 58 & 59 & 61 & 63 & 65 & 41 & & \\
\hline & Escaped Rate & 1 & 1 & 1 & 1 & 1 & 1 & 5 & & \\
\hline & Subgroup Length & \multicolumn{6}{|c|}{28} & 15 & & \\
\hline
\end{tabular}

Table 4. Escaped Tests Comparison for Circuit 3

\begin{tabular}{|c|c|c|c|c|c|c|c|c|c|c|c|c|c|c|c|c|c|c|c|}
\hline \multirow{3}{*}{ Reference [12] } & Test No. & 63 & 71 & 137 & 185 & $188 \mid 1$ & 193 & 261 & 194 & 262 & & & & & & & & & \\
\hline & Escaped Rate & 1 & 1 & 1 & 1 & 1 & 1 & 5 & 1 & 3 & & & & & & & & & \\
\hline & Subgroup Length & 4 & \multicolumn{6}{|c|}{118} & 24 & 65 & & & & & & 1 & & & \\
\hline & Test No. & 73 & 75 & 77 & 79 & 81 & 84 & 86 & 88 & 90 & 92 & 136 & 137 & 152 & 171 & 184 & 185 & 188 & 193 \\
\hline \multirow[t]{2}{*}{ Proposed } & Escaped Rate & 1 & 1 & 1 & 1 & \begin{tabular}{l|l}
1 \\
\end{tabular} & 1 & 1 & 1 & 1 & 1 & 1 & 1 & 1 & 1 & \begin{tabular}{|l|}
1 \\
\end{tabular} & \begin{tabular}{|c|}
1 \\
\end{tabular} & 1 & 1 \\
\hline & Subgroup Length & \multicolumn{9}{|c|}{12} & \multicolumn{9}{|c|}{118} \\
\hline
\end{tabular}

\subsection{Wireless Circuit with 70 Specifications}

The second industry data set has $50 \mathrm{~K}$ samples with 70 specifications. Again, $2 \mathrm{~K}$ samples are selected as the training set and the $48 \mathrm{~K}$ remaining samples are used to compute the DPPM and the Number of Tests. Table 1 shows that the proposed method has about five times fewer DPPM and less Number of Tests than the method in Reference [12].

Table 3 shows a breakdown of escaped tests for the two methods. In this case, the proposed method does not result in any escapes due to tests 46 and 68, and fewer escapes due to the rest of the tests in this list.

\subsection{Large-Scale Mixed-Signal Circuit}

We apply the two methods to a production data set of a large scale circuit, which has $50 \mathrm{~K}$ samples with 264 specifications. The first $2 \mathrm{~K}$ samples are selected as the training set, and the rest of the data are used to compute the DPPM and the Number of Tests. Table 1 shows that the proposed method reduces both Number of Tests and DPPM compared to the baseline [12].

Table 4 shows the escaped tests and how many faulty circuits escaped for both methods. The proposed method has fewer tests where at least one faulty device escaped.

And for this largest-scale circuit in our simulations, the total computation time for each test including the ones for Pfail(Tj) and the JPDF is less than 1ms on a 4.2GHz/64GB RAM PC.

\subsection{EEPROM Readout Circuit with 57 Selected Specifications}

The final circuit is an EEPROM readout circuit with 57 analog specifications and 1,002K samples. Similarly, 2K samples are selected as the training set and the rest of the data are used to compute the DPPM and the Number of Tests. Table 1 shows that the proposed method results in fewer DPPM and less test time compared to Reference [12].

Table 5 shows the escaped tests for the two methods. The results have the similar pattern as circuit 3 that tests in subgroups with longer length escaped more in Reference [12] than in the proposed method. Note that test 40 escaped for many more samples in the proposed than in [12]. 
Table 5. Escaped Tests Comparison for Circuit 4

\begin{tabular}{|c|c|c|c|c|c|c|c|c|c|c|c|c|c|c|c|c|c|}
\hline \multirow{3}{*}{ Reference [12] } & Test No. & 40 & 24 & 27 & 28 & 30 & 34 & 35 & 37 & 43 & 45 & 47 & 49 & 50 & 52 & 54 & 56 \\
\hline & Escaped Rate & 12 & 1 & 3 & 4 & 3 & 1 & 5 & 1 & 40 & 42 & 45 & 44 & 45 & 43 & 51 & 45 \\
\hline & Subgroup Length & 10 & \multicolumn{15}{|c|}{ 4 } \\
\hline \multirow{3}{*}{ Proposed } & Test No. & 40 & 23 & 24 & 27 & 28 & 29 & 30 & 31 & 32 & 34 & 35 & 37 & 43 & 51 & & \\
\hline & Escaped Rate & 47 & 2 & 1 & 4 & 4 & 5 & 1 & 7 & 6 & 1 & 5 & 1 & 1 & 1 & & \\
\hline & Subgroup Length & 10 & \multicolumn{13}{|c|}{46} & & \\
\hline
\end{tabular}

This test has no fail samples in the training set, hence leading it to be placed towards the end of the test list according to the marginality condition but randomly placed in [12].

\section{CONCLUSION}

In this work, the fail rate, the failing likelihood, and the higher- order correlations among the specifications are considered simultaneously for initial test list ordering. The strategy enables us to apply the test with information as much as possible one-by-one during the test process. Integrated with the proposed initial test list, the test method in Reference [12] gives better DPPM and Number of Tests. In the ordering strategy, an information-concerned search method is applied, in which failed tests or tests likely to fail are placed in an information concern, which is different from the previous works on initial test list ordering. Thus, some tests with low fail rate have more chance to be placed at the front in the initial test list and give better test quality and test speed in the results. However, we do not have the precise test execution time included in the circuit data. Certainly, if this information were available, it should be considered in the optimization flow in the future work.

\section{ACKNOWLEDGMENTS}

This work is supported by National Science Foundation with Grant Number 1617562 and by Semiconductor Research Corporation by Task Number 2712.003.

\section{REFERENCES}

[1] P. Drineas and Y. Makris. 2003. Independent test sequence compaction through integer programming. In Proceedings of the IEEE International Conference on Computer Design. 380-386.

[2] H.-G. Stratigopoulos, P. Drineas, M. Slamani, and Y. Makris. 2007. Non-RF to RF test correlation using learning machines: A case study. In Proceedings of the IEEE VLSI Test Symposium. 9-14.

[3] S. Biswas and R. D. Blanton. 2008. Test compaction for mixed-signal circuits using pass-fail test data. In Proceedings of the IEEE VLSI Test Symposium. 299-308.

[4] S. Biswas and R. D. Blanton. 2006. Statistical test compaction using binary decision trees. IEEE Design Test Comput. 23, 6 (2006), 452-462.

[5] S. Benner and O. Boroffice. 2001. Optimal production test times through adaptive test programming. In Proceedings of the IEEE International Test Conference. 908-915.

[6] Christian Stritwieser. 2015. Real-time adaptive test algorithm including test escape estimation method. In Proceedings of the 20 th International Mixed-Signal Testing Workshop (IMSTW'15). 1-6.

[7] A. Ahmadi, A. Nahar, B. Orr, M. Past, and Y. Makris. 2016. Wafer-level process variation-driven probe-test flow selection for test cost reduction in analog/RF ICs. In Proceedings of the IEEE VLSI Test Symposium. 1-6.

[8] H.-G. Stratigopoulos and Christian Streiwieser. 2017. Adaptive test flow for mixed-signal ICs. In Proceedings of the IEEE VLSI Test Symposium. 1-6.

[9] E. Yilmaz and S. Ozev. 2009. Adaptive test elimination for analog/RF circuits. In Proceedings of the IEEE Design Automation Conference. 720-725.

[10] E. Yilmaz, S. Ozev, and K. M. Butler. 2010. Adaptive test flow for mixed-signal/RF circuits using learned information from device under test. In Proceedings of the IEEE International Test Conference. 1-10. 
[11] E. Yilmaz, S. Ozev, and K. M. Butler. 2013. Per-device adaptive test for analog/RF circuits using entropy-based process monitoring. IEEE Trans. VLSI Syst. 21, 6 (2013), 1116-1128.

[12] E. Yilmaz, S. Ozev, and K. M. Butler. 2011. Adaptive multidimensional outlier analysis for analog and mixed signal circuits. In Proceedings of the IEEE International Test Conference. 1-8.

[13] T. H. Cormen, C. Stein, R. L. Rivest, and C. E. Leiserson. 2001. Introduction to Algorithms (2nd. ed.). McGraw-Hill Higher Education.

[14] Suraj Sindia and Vishwani D. Agrawal. 2014. Specification test minimization for given defect level. In Proceedings of the 15 th Latin American Test Workshop. 1-6.

[15] L. Milor. 1998. A tutorial introduction to research on analog and mixed-signal circuit testing. IEEE Trans. Circ. Syst. II: Analog Digital Sig. Proc. 45, 10 (1998), 1389-1407.

[16] S. D. Huss and R. S. Gyurcsik. 1991. Optimal ordering of analog integrated circuit tests to minimize test time. In Proceedings of the IEEE/ACM Design Automation Conference. 494-499.

[17] W. Jiang and B. Vinnakota. 1999. Defect-oriented test scheduling. In Proceedings of the IEEE VLSI Test Symposium. 433-438.

[18] Baris Arslan and Alex Orailoglu. 2016. Aggressive test cost reductions through continuous test effectiveness assessment. IEEE Trans. Comput.-Aided Design Integr. Circ. Syst. 35, 12 (2016), 2093-2103.

[19] S. Biswas, P. Li, R. D. Blanton, and L. T. Pileggi. 2005. Specification test compaction for analog circuits and MEMS [accelerometer and opamp examples]. In Proceedings of the IEEE Design, Automation and Test in Europe. 164-169.

[20] S. S. Akbay, J. L. Torres, J. M. Rumer, A. Chatterjee, and J. Amtsfield. 2006. Alternate test of RF front ends with IP constraints: Frequency domain test generation and validation. In Proceedings of the IEEE International Test Conference. 1-10.

[21] N. Kupp, P. Drineas, M. Slamani, and Y. Makris. 2009. On boosting the accuracy of non-RF to RF correlation-based specification test compaction. Springer f. Electron. Test. 25, 6 (2009), 309-321.

[22] M. Chen and A. Orailoglu. 2008. Test cost minimization through adaptive test development. In Proceedings of the IEEE International Conference on Computer Design. 234-239.

[23] K. Huang, J. Wen, and J. Willmore. 2016. Test-suite-based analog/RF test time reduction using canonical correlation. IEEE Trans. Comput.-Aided Design Integr. Circ. Syst. 35, 12 (2016), 2143-2147.

[24] Thomas M. Cover and A. Joy. 2006. Element of Information Theory (2nd. ed.). Wiley-Interscience, New York, NY, $19-20$.

[25] Gene H. Golub and Charles F. Van Loan. Matrix Computations (4th. ed.). JHU Press, Baltimore, MD, 246-259.

Received August 2018; revised December 2018; accepted January 2019 